\title{
Tratamento Percutâneo de Pseudoaneurismas por Injeção de Trombina Guiada por Ultrassom
}

\author{
Silvio Gioppato 1 , Alexandre Munhoz ${ }^{2}$, Marcos Marins², Thomas Borges Conforti', \\ Hélio J. Castello Jr. ${ }^{1}$, Marcelo J. C. Cantarelli', Guilherme Ferragut Attizzani ${ }^{1}$
}

\section{RESUMO}

Introdução: Pseudoaneurisma iatrogênico ocorre em 0,5\% a $2 \%$ dos procedimentos percutâneos diagnósticos e em até $7 \%$ a $8 \%$ dos procedimentos percutâneos terapêuticos. A oclusão do pseudoaneurisma pela injeção de trombina guiada por ultrassom diretamente no interior do saco aneurismático é técnica pouco invasiva, de execução rápida e segura, e com alto índice de sucesso. A proposta deste trabalho é relatar nossa experiência no tratamento do pseudoaneurisma iatrogênico por injeção de trombina guiada por ultrassom. Método: Entre setembro de 2006 e fevereiro de 2010 realizamos 1.811 procedimentos cardíacos percutâneos com ocorrência de 15 pseudoaneurismas $(0,8 \%)$, dos quais 13 foram tratados com injeção de trombina guiada por ultrassom de forma consecutiva. Características clínicas, tipo de procedimento, medicações coadjuvantes, tamanho e morfologia dos pseudoaneurismas, dose de trombina utilizada, resultado da terapia e ocorrência de complicações foram documentados e armazenados prospectivamente em banco de dados e, então, analisados retrospectivamente. Resultados: A taxa de sucesso primário foi de $100 \%$, com apenas uma recidiva sete dias após a abordagem inicial, tratada com sucesso pela mesma técnica. Não foi observada ocorrência de complicações relacionadas à injeção de trombina guiada por ultrassom e todos os pacientes apresentavam-se assintomáticos aos 30 dias de acompanhamento. Conclusão: A injeção de trombina guiada por ultrassom realizada por profissionais com experiência em ultrassom vascular e em punções transparietais de cistos e coleções é uma opção terapêutica viável, prática e segura no tratamento do pseudoaneurisma iatrogênico.

DESCRITORES: Trombina. Cateterismo cardíaco. Ultrassonografia de intervenção.

\footnotetext{
1 Serviço de Hemodinâmica - Hospital Vera Cruz - Campinas, SP, Brasil.

${ }^{2}$ Serviço de Radiologia e Ultrassonografia - Hospital Vera Cruz Campinas, SP, Brasil.

Correspondência: Silvio Gioppato. Rua Onze de Agosto, 495 -

Centro - Campinas, SP, Brasil - CEP 13013-100

E-mail: gioppato@terra.com.br

Recebido em: 18/3/2010 • Aceito em: 26/5/2010
}

\section{ABSTRACT \\ Percutaneous Treatment of Pseudoaneurysms by Ultrasound-guided Thrombin Injection}

Background: latrogenic pseudoaneurysm is observed in $0.5 \%$ to $2 \%$ of diagnostic percutaneous procedures and in up to $7 \%$ to $8 \%$ of therapeutic percutaneous procedures. Pseudoaneurysm occlusion by ultrasound-guided thrombin injection directly in the aneurysm sac is a minimally invasive, quick and safe procedure with a high success rate. The aim of our study is to report our initial experience in treatment of iatrogenic pseudoaneurysms by ultrasound-guided thrombin injection. Method: Between September 2006 and February 2010, 1,811 percutaneous coronary interventions were carried out with an occurrence of 15 pseudoaneurysms $(0.8 \%)$, of which 13 were consecutively treated by ultrasound-guided thrombin injection. Clinical characteristics, type of percutaneous procedure, adjuvant therapy, size and morphology of pseudoaneurysms, thrombin dose, success and complication rate were prospectively documented and later retrospectively analyzed. Results: Primary success rate was $100 \%$ with just one recurrence seven days after the initial approach, which was successfully treated using the same technique. There were no complications related to the ultrasound-guided thrombin injection and all of the patients were asymptomatic 30 days after the procedure. Conclusion: Ultrasound-guided thrombin injection performed by professionals experienced in intravascular ultrasound and transparietal punctures of cysts and collections is a feasible, practical and safe option in the treatment of iatrogenic pseudoaneurysms.

KEY-WORDS: Thrombin. Heart catheterization. Ultrasonograpy, interventional.

$\mathbf{P}$ seudoaneurisma iatrogênico é uma das possíveis complicações decorrentes de procedimentos endovasculares com incidência de $0,5 \%$ a $2 \%$ nos procedimentos diagnósticos e em até 7\% a $8 \%$ nos procedimentos terapêuticos. ${ }^{1,2}$

Tradicionalmente o reparo cirúrgico e a compressão guiada por ultrassom com Doppler são as técnicas corretivas mais difundidas. A correção cirúrgica é o mais antigo método de tratamento que, por sua pró- 
pria natureza, envolve inúmeras desvantagens, com significante parcela dos pacientes apresentando complicações como infecções e deiscência de sutura ou formação de fístulas linfáticas. ${ }^{3-5}$ A compressão guiada por ultrassom com Doppler tem sucesso em torno de 90\% e, por ser não-invasiva, elimina diversos inconvenientes do reparo cirúrgico; no entanto, nem todos os pacientes toleram o longo tempo de compressão necessário para obter a trombose, às vezes extremamente dolorosa, além de apresentar taxa de insucesso em cerca de dois terços dos pacientes em uso de anticoagulantes. ${ }^{6}$

Uma abordagem mais recente é a oclusão do pseudoaneurisma pela injeção de trombina guiada por ultrassom diretamente no interior do saco aneurismático. A injeção percutânea de trombina foi descrita pela primeira vez por Cope e Zeit ${ }^{7}$, em 1986, que realizaram o procedimento guiado por fluoroscopia. Mais tarde, Kang et al. ${ }^{8}$ descreveram a técnica como aplicada hoje e, desde então, o número de publicações referentes ao tema vem aumentando progressivamente com elevados índices de sucesso. ${ }^{9-13}$ A natureza pouco invasiva, a execução rápida e segura, dispensando a necessidade de anestesia ou analgesia potente, e os altos índices de sucesso tornaram a injeção de trombina guiada por ultrassom uma boa alternativa para o tratamento dos pacientes com pseudoaneurisma iatrogênico, principalmente nos que apresentam elevado risco para o reparo cirúrgico ou risco de insucesso com compressão guiada por ultrassom com Doppler, como idosos, obesos e portadores de pseudoaneurismas iatrogênicos volumosos e/ou de morfologia complexa.

A proposta deste trabalho é relatar nossa experiência no tratamento do pseudoaneurisma iatrogênico por injeção de trombina guiada por ultrassom.

\section{MÉTODO}

\section{Pacientes}

Entre setembro de 2007 e 1ㅇ de março de 2010, foram realizados em nosso serviço 1.811 procedimentos cardíacos entre exames diagnósticos e intervenções terapêuticas, com ocorrência de 15 pseudoaneurismas $(0,8 \%)$, sendo 14 de artéria femoral e 1 de artéria braquial.

A população em estudo é composta predominantemente por pacientes do sexo masculino (67\%), com idade variando de 52 a 84 anos (mediana de 76 anos), com índice de massa corporal variando de 20 a 43 (média de 29,5), dos quais 100\% (15/15) eram hipertensos, 53\% (8/15) eram diabéticos, 47\% (7/15) tinham história de tabagismo, 40\% (6/15) tinham sido submetidos a revascularização miocárdica prévia, 47\% (7/15) eram portadores de insuficiência renal crônica, e $47 \%(7 / 15)$ eram portadores de doença arterial periférica. O diagnóstico de síndrome coronária aguda
(SCA), com ou sem supradesnivelamento do segmento ST, foi a apresentação clínica predominante $(67 \% ; 10 / 15)$, $73 \%$ dos casos (11/15) estavam em uso de dupla antiagregação plaquetária com aspirina e clopidogrel, e a combinação de aspirina + clopidogrel + inibidor da glicoproteína Ilb/IIla era utilizada por $20 \%$ dos pacientes (3/15). O uso de heparina de baixo peso molecular, em dose plena ou profilática, esteve presente em $60 \%$ dos casos (9/15), em um único caso houve associação com terapia trombolítica e dois pacientes estavam em uso de anticoagulantes orais (Tabela 1).

Dos 15 casos, 2 não receberam injeção de trombina por se tratar de pacientes multiarteriais com indicação de revascularização miocárdica cirúrgica, optando-se, nesses casos, pela correção do pseudoaneurisma a céu aberto no ato cirúrgico, servindo como mera comparação evolutiva com o grupo que recebeu tratamento com injeção de trombina.

\section{Procedimento}

Todos os procedimentos foram realizados no departamento de ultrassonografia do Hospital Vera Cruz, em Campinas (SP), por profissionais com larga experiência na técnica do ultrassom vascular e de punção

TABELA 1

Características clínicas

\begin{tabular}{|c|c|}
\hline Total, $\mathrm{n}$ & 15 \\
\hline Sexo masculino, n (\%) & $10(66,7)$ \\
\hline Idade, anos (mediana, min.-máx.) & $76(52-84)$ \\
\hline IMC, kg/m² (média \pm DP) & $28,4 \pm 5$ \\
\hline Hipertensão, n (\%) & $15(100)$ \\
\hline Diabetes melito, n (\%) & $8(53)$ \\
\hline História de tabagismo, n (\%) & $7(47)$ \\
\hline Revascularização do miocárdio, n (\%) & $6(40)$ \\
\hline Insuficiência renal crônica, n (\%) & $7(47)$ \\
\hline Doença arterial periférica, n (\%) & $7(47)$ \\
\hline $\begin{array}{l}\text { SCA com ou sem } \\
\text { supradesnivelamento de ST, n (\%) }\end{array}$ & $10(66,7)$ \\
\hline \multicolumn{2}{|l|}{ Tratamento farmacológico, n (\%) } \\
\hline Aspirina + clopidogrel & $11(73)$ \\
\hline Aspirina + clopidogrel + IGP IIb/IIIa & $3(20)$ \\
\hline Antitrombínicos (HBPM ou HNF) & $9(60)$ \\
\hline Anticoagulante oral & $2(13)$ \\
\hline Trombolítico & $1(6,7)$ \\
\hline \multicolumn{2}{|l|}{ Procedimento, n (\%) } \\
\hline Cateterismo diagnóstico & $5(33,3)$ \\
\hline Intervenção percutânea & $10(66,7)$ \\
\hline
\end{tabular}


transparietal de cistos e coleções em diferentes territórios, tendo sido utilizado equipamento Philips HDI 5.000 (Washington, Estados Unidos) com transdutores de banda larga (lineares, $5 \mathrm{MHz}$ a $12 \mathrm{MHz}$; e convexos, $2 \mathrm{MHz}$ a $5 \mathrm{MHz}$ ).

O preparado comercial de trombina utilizado foi o Beriplast ${ }^{\circledR}$ (Aventis Behring, Alemanha), que consiste em dois componentes: trombina humana liofilizada (400 Ul/ml a $600 \mathrm{Ul} / \mathrm{ml}$ ) reconstituída em solução de cloreto de cálcio (preparado 1), e fibrinogênio humano e fator XIII, reconstituídos em solução de aprotinina bovina (preparado 2).

Os dois preparados, quando utilizados simultaneamente, formam um adesivo elástico empregado como agente hemostático em cirurgias com grandes áreas cruentas ou com áreas de anastomose vascular, principalmente em situações de depleção dos fatores da coagulação. Como na nossa amostra o objetivo foi selar uma cavidade paravascular repleta de sangue constitucionalmente íntegro, optamos por utilizar apenas o preparado de trombina humana, já que há relatos na literatura de fenômenos alérgicos relacionados aos componentes de origem bovina.

\section{Técnica}

Um minucioso estudo ultrassonográfico preliminar foi realizado para aferir medidas e morfologia do saco aneurismático, tamanho e posição do orifício comunicante, bem como conhecer a direção preferencial dos fluxos de entrada e saída no interior do pseudoaneurisma a fim de traçar a melhor estratégia para cada caso.

Registro do padrão das curvas com Doppler nas artérias femorais, superficial e profunda, foi realizado antes e após a injeção de trombina para garantir ausência de complicação embólica.

Adotou-se a técnica de abordar o pseudoaneurisma, sempre que possível, pelo pólo mais distante do orifício comunicante, preferindo a região com predomínio do fluxo de entrada, a fim de minimizar o risco de embolia arterial. O preparo da pele na região inguinal foi feito com solução alcoólica de clorexidina a $2 \%$ e o local foi posteriormente coberto com campo cirúrgico estéril. O transdutor de ultrassom foi inserido em saco plástico estéril e infiltração anestésica no local da punção foi feita com solução de xilocaína a $2 \%$.

O conteúdo do frasco com liofilizado de trombina humana foi reconstituído em solução de cloreto de cálcio, conforme recomendação do fabricante, e aspirado em seringa de $1 \mathrm{ml}$ fornecida no kit do produto. Guiada por ultrassom bidimensional, uma agulha $22 \mathrm{G}$ foi introduzida no interior do pseudoaneurisma, segundo a técnica descrita, através da qual injetamos pequenas quantidades da solução de trombina, acompanhando as modificações do padrão do fluxo no interior do pseudoaneurisma por meio de Doppler em cores até que ocorresse trombose completa da cavidade e o consequente desaparecimento do fluxo em seu interior. O processo ocorre de modo muito rápido, com formação de trombo imediatamente após a injeção do conteúdo da seringa, como pode ser observado na Figura 1.

\section{Definições}

- Sucesso primário: obtenção do completo fechamento do pseudoaneurisma na primeira abordagem terapêutica, independentemente do volume de trombina necessário.

- Sucesso secundário: quando uma segunda injeção de trombina foi necessária para tratar recidiva do pseudoaneurisma, definida como recorrência após 24 horas ou mais de um sucesso primário.

- Insucesso: quando outra técnica de oclusão foi necessária para completar o tratamento, tanto por reparo cirúrgico como por compressão guiada por ultrassom.

- Recidiva: recorrência do pseudoaneurisma 24 horas ou mais após um sucesso primário.

\section{RESULTADOS}

O intervalo entre o procedimento desencadeador e o diagnóstico do pseudoaneurisma variou de dois a oito dias em todos os casos, à exceção de um caso em que se manifestou tardiamente (depois de mais de 90 dias da intervenção índice) em paciente portador de fibrilação atrial crônica e que estava em uso de anticoagulação oral e antiagregação plaquetária com aspirina. Sucesso primário foi alcançado em todos os casos tratados $(100 \% ; 13 / 13)$, com apenas uma recidiva $(7,7 \% ; 1 / 13)$ sete dias após o sucesso primário de um pseudoaneurisma de morfologia complexa (lobulado) tratado por reinjeção de trombina guiada por ultrassom com sucesso secundário. A dose de trombina necessária para obtenção da trombose completa dos pseudoaneurismas variou de $300 \mathrm{UI}$ a 1.000 UI, não havendo ocorrência de complicação diretamente relacionada à injeção de trombina. Todos os pacientes tratados com injeção de trombina apresentavam-se assintomáticos no seguimento de 30 dias. Nos dois casos submetidos a correção cirúrgica, um teve boa evolução, sem qualquer intercorrência, e o outro evoluiu com infecção local e deiscência da sutura, necessitando tratamento prolongado com antibióticos e cicatrização por segunda intenção, porém atualmente encontra-se assintomático. Esses dados estão apresentados, de forma resumida, na Tabela 2 .

\section{DISCUSSÃO}

Pseudoaneurisma iatrogênico após cateterização cardíaca caracteriza-se por solução de continuidade na parede arterial induzida pela agulha de punção ou pela bainha introdutora. Isso permite que o sangue 

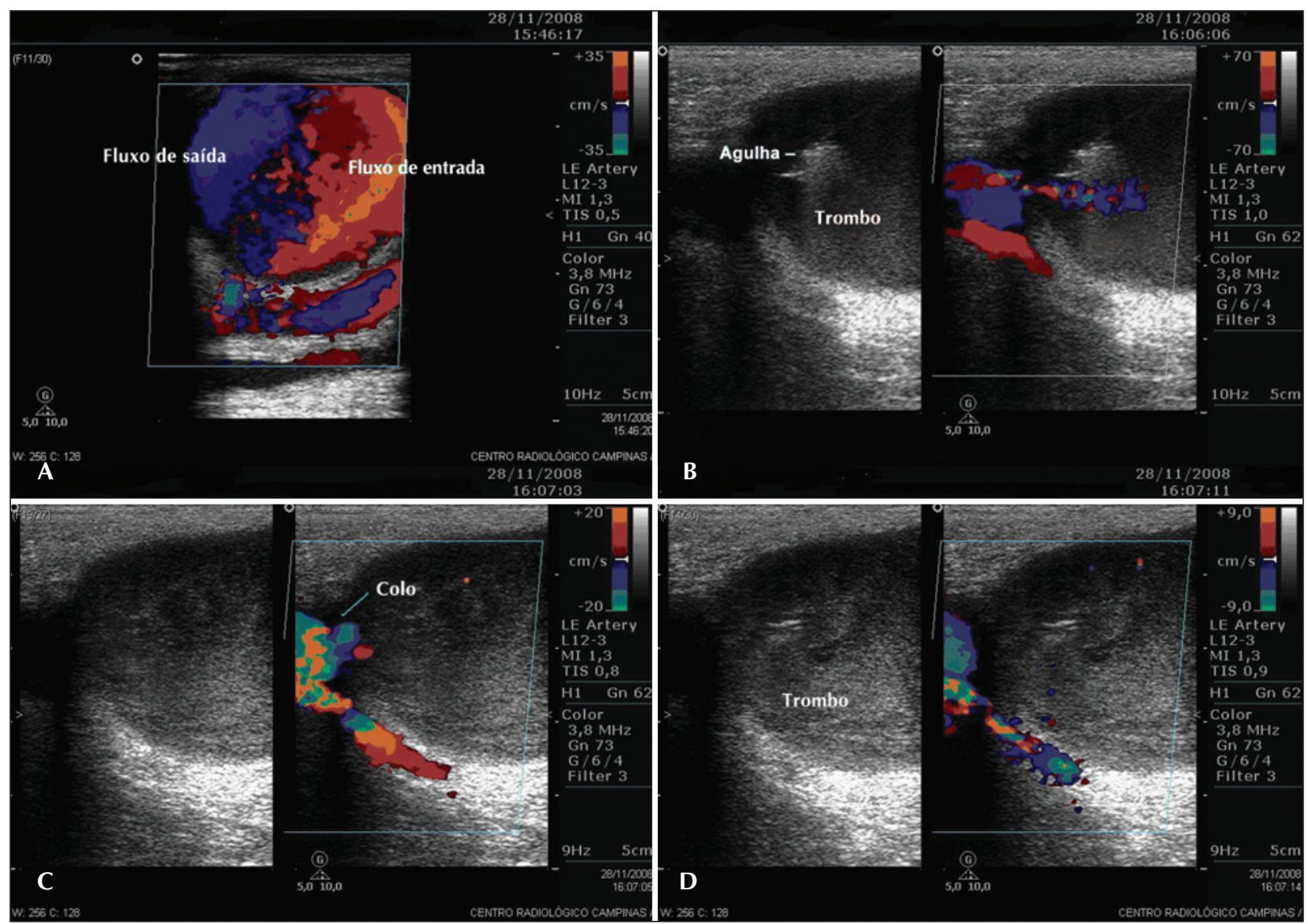

Figura 1 - Sequência de imagens demonstrando processo de trombose do pseudoaneurisma iatrogênico pela injeção de trombina. Em A, Doppler em cores demonstrando grande pseudoaneurisma $(48 \mathrm{cc})$, com típico padrão yin-yang dos fluxos de entrada e saída de sangue. Em B, fase inicial da trombose com massa de trombo predominante na via de entrada e junto à ponta da agulha. Em $\mathrm{C}$, fase final da trombose com saco aneurismático trombosado, restando apenas o colo. Em D, processo concluído, não havendo qualquer evidência de fluxo ao Doppler no interior do pseudoaneurisma iatrogênico.

extravase da luz vascular para além dos limites do vaso, dissecando as estruturas perivasculares e dando origem a um saco aneurismático cuja parede pode ser constituída pelas camadas média e adventícia do próprio vaso ou apenas pelas estruturas adjacentes. Dependendo de inúmeros fatores, os pseudoaneurismas podem evoluir para trombose espontânea ou progredir com desenvolvimento de complicações potencialmente graves, como infecção local, compressão de estruturas neurovasculares adjacentes ou ruptura.

As opções terapêuticas possíveis para correção de pseudoaneurisma são o reparo cirúrgico, a compressão guiada por ultrassom, a oclusão percutânea com implante de stent recoberto, e a injeção percutânea de trombina no interior do pseudoaneurisma guiada por ultrassom. Desde que foi descrita por Fellmeth et al. ${ }^{14}$, em 1991, a compressão guiada por ultrassom com Doppler mostrou-se tão eficaz quanto o reparo cirúrgico, porém com inúmeras vantagens que a tornaram, a partir de então, a opção de escolha no tratamento dos pseudoaneurismas. Entretanto, essa técni- ca tem como limitantes o tempo prolongado de compressão necessário para obter a trombose e o desconforto, às vezes intolerável, ao paciente, responsáveis pelo menor índice de sucesso naqueles com grandes pseudoaneurismas, nos anticoagulados ou com dupla antiagregação plaquetária, com falha terapêutica variando de $15 \%$ a $38 \%$ e índices de recidiva após o sucesso inicial de $20 \%$ a $30 \%$ em pacientes recebendo terapia anticoagulante. ${ }^{6}$

Comparada à compressão guiada por ultrassom com Doppler, a injeção de trombina tem índices de sucesso superiores, chegando a $96 \%$ contra $74 \%$, como no estudo de Paulson et al. ${ }^{15}$, em que a proporção de pacientes com terapia anticoagulante foi significativamente maior no grupo trombina que no grupo compressão. Reforçando a superioridade da injeção de trombina guiada por ultrassom sobre a compressão guiada por ultrassom com Doppler, Brophy et al. ${ }^{16}$ trataram com injeção de trombina 15 pacientes em que a terapia de compressão havia falhado, obtendo sucesso em todos a despeito do regime de anticoagulação. Em outro 
TABELA 2

Características físicas e morfológicas dos pseudoaneurismas iatrogênicos, técnica de abordagem, sucesso primário, recidiva e complicações

\begin{tabular}{|c|c|c|c|c|c|c|c|c|}
\hline Caso & $\begin{array}{c}\text { Intervalo } \\
\text { P/D } \\
\text { (dias) }\end{array}$ & $\begin{array}{c}\text { Dimensões } \\
(\mathbf{c m})\end{array}$ & $\begin{array}{c}\text { Volume } \\
\text { do PSA } \\
\text { (cc) }\end{array}$ & Morfologia & Abordagem & $\begin{array}{l}\text { Sucesso } \\
\text { primário }\end{array}$ & Recidiva & Complicação \\
\hline 1 & 4 & $7,2 \times 2,7 \times 2,5$ & 25,3 & Simples & ITGUS & Sim & Não & Não \\
\hline 2 & 3 & $2,8 \times 3,3 \times 1,8$ & 8,6 & Simples & ITGUS & Sim & Não & Não \\
\hline 3 & 3 & $3,3 \times 3,2 \times 2,2$ & 12,1 & Simples & ITGUS & Sim & Não & Não \\
\hline 4 & 5 & $3,2 \times 1,0 \times 1,7$ & 2,8 & Simples & ITGUS & Sim & Não & Não \\
\hline 5 & 4 & $5,2 \times 2,3 \times 1,6$ & 10,2 & Lobulado & ITGUS & Sim & Sim & Não \\
\hline 6 & 6 & $2,1 \times 1,7 \times 1,2$ & 2,2 & Simples & ITGUS & Sim & Não & Não \\
\hline 7 & 7 & $4,6 \times 5,9 \times 3,4$ & 48 & Simples & ITGUS & Sim & Não & Não \\
\hline 8 & 5 & $3,5 \times 4,1 \times 2,7$ & 20,1 & Simples & ITGUS & Sim & Não & Não \\
\hline 9 & 3 & $2,7 \times 1,9 \times 2,9$ & 7,7 & Lobulado & ITGUS & Sim & Não & Não \\
\hline 10 & 3 & $5,5 \times 3,5 \times 1,4$ & 31 & Simples & ITGUS & Sim & Não & Não \\
\hline 11 & 7 & $1,3 \times 1,5 \times 2,7$ & 2,7 & Simples & ITGUS & Sim & Não & Não \\
\hline 12 & 4 & $3,0 \times 2,5 \times 1,9$ & 7,4 & Simples & ITGUS & Sim & Não & Não \\
\hline 13 & 95 & $3,0 \times 2,1 \times 3,1$ & 10,2 & Simples & ITGUS & Sim & Não & Não \\
\hline 14 & 7 & $3,1 \times 1,8 \times 2,1$ & 6,1 & Simples & Cirurgia & - & - & Sim \\
\hline 15 & 8 & $2,9 \times 2,4 \times 2,5$ & 9,0 & Simples & Cirurgia & - & - & Não \\
\hline
\end{tabular}

Intervalo $\mathrm{P} / \mathrm{D}$ = intervalo entre o procedimento desencadeador e o diagnóstico; ITGUS = injeção de trombina guiada por ultrassom; PSA = pseudoaneurisma iatrogênico.

estudo com 240 pacientes, Krueger et al. ${ }^{10}$ obtiveram sucesso primário em 93,8\% dos casos, sendo 95,8\% nos pseudoaneurismas simples e $89 \%$ nos complexos. Já o índice de sucesso secundário foi de 99,6\%, sendo $100 \%$ nos casos simples e $99 \%$ para os complexos, demonstrando que a geometria do pseudoaneurisma influencia de maneira significativa as taxas de sucesso. Os autores também sugerem que a técnica de injeção interfere no resultado, orientando que se inicie a injeção da trombina pelo lobo distal do pseudoaneurisma, pois nos poucos casos em que ocorreu reperfusão tardia ( $>24$ horas) de um ou mais lobos do pseudoaneurisma a injeção havia sido realizada no lobo proximal.

Complicações relacionadas com a injeção de trombina parecem ocorrer em baixa frequência, com relatos de casos isolados de infecção local, trombose e/ou tromboembolismo arterial e reação alérgica à trombina. Das complicações conhecidas na literatura as mais temidas são a trombose e/ou o tromboembolismo arterial, que podem ser prevenidos evitando a injeção de trombina próxima ou no próprio colo do pseudoaneurisma. ${ }^{4,6,11}$

Nossa população exibe perfil clínico de grande complexidade, formada principalmente por pacientes idosos, obesos, vítimas de síndromes coronárias agudas, submetidos a procedimentos terapêuticos em que, sabidamente, os cateteres e introdutores são de maior calibre e a combinação de antiagregantes, antitrombínicos e, às vezes, trombolíticos aumenta muito o risco de desenvolvimento de pseudoaneurisma iatrogênico, bem como o insucesso e as complicações de seu tratamento, por meio tanto da cirurgia como da compressão guiada por ultrassom com Doppler. Além da complexidade clínica, nossa amostra também se mostrou complexa do ponto de vista anatômico, com pseudoaneurismas volumosos (média de 13,6 cc), os quais exibem menor índice de sucesso pela técnica de compressão.

Obtivemos sucesso primário em 100\% dos casos, com apenas uma recidiva, sem nenhum tipo de complicação relacionada ao procedimento. Ao contrário dos dois casos encaminhados para tratamento cirúrgico, dos quais apenas um teve bom resultado, já que o outro evoluiu com complicação infecciosa que necessitou reintervenções e antibioticoterapia prolongada.

Por ser uma análise observacional pós-hoc de dados compilados prospectivamente, sem grupo controle e com pequeno número de pacientes, este estudo apresenta forte limitação quanto ao peso de suas conclusões, servindo, mormente, para demonstrar a experiência inicial de um grupo.

\section{CONCLUSÃO}

Nossos dados demonstram que a injeção de trombina guiada por ultrassom é uma opção viável, prática 
e segura para o tratamento de pseudoaneurisma iatrogênico pós-cateterização arterial. Ressaltamos que a cuidadosa análise das características anatômicas do pseudoaneurisma e o rigor nas técnicas de punção e injeção da trombina concorrem para um bom resultado final.

\section{CONFLITO DE INTERESSES}

Os autores declararam inexistência de conflito de interesses relacionado a este manuscrito.

\section{REFERÊNCIAS}

1. Katzenschlager R, Ugurlouglu A, Ahmadi A, Hülsmann M, Koppensteiner R, Larch E, et al. Incidence of pseudoaneurysm after diagnostic and therapeutic angiography. Radiology. 1995; 195(2):463-6.

2. Wixon CL, Philpott JM, Bogey WM Jr, Powell CS. Duplexdirected thrombin injection as method to treat femoral artery pseudoaneurysms. J Am Coll Surg. 1998;187(4):464-6.

3. Zarge J, Villemure P, Mathewson C, Lawrence J. Complications related to thrombin injection for pseudoaneurysm repair. J Vasc Tech. 2001;25:209-12.

4. Ricci MA, Trevisani GT, Pilcher DB. Vascular complications of cardiac catheterization. Am J Surg. 1994;167(4):375-8.

5. San Norberto García EM, González-Fajardo JA, Gutiérrez V, Carrera S, Vaquero C. Femoral pseudoaneurysms post-cardiac catheterization surgically treated: evolution and prognosis. Interact Cardiovasc Thorac Surg. 2009;8(3):353-8.

6. Saad NE, Saad WE, Davies MG, Waldman DL, Fultz PJ, Rubens DJ. Pseudoaneurysms and the role of minimally invasive techniques in their management. Radiographics. 2005; 25 Suppl 1:S173-89.

7. Cope C, Zeit R. Coagulation of aneurysms by direct percutaneous thrombin injection. AJR Am J Roentgenol. 1986; 147(2):383-7.
8. Kang SS, Labropoulos N, Mansour MA, Baker WH. Percutaneous ultrasound guided thrombin injection: a new method for treating postcatheterization femoral pseudoaneurysms. J Vasc Surg. 1998;27(6):1032-8.

9. Kang SS, Labropoulos N, Mansour MA, Michelini M, Filliung D, Baubly MP, et al. Expanded indications for ultrasoundguided thrombin injection of pseudoaneurysms. J Vasc Surg. 2000;31(2):289-8.

10. Krueger K, Zaehringer M, Strohe D, Stuetzer H, Boecker J, Lackner K. Postcatheterization pseudoaneurysm: results of US-guided percutaneous thrombin injection in 240 patients. Radiology. 2005;236(3):1104-10.

11. Pinto DM, Dias Junior JO, Fonseca BLC, Moreialvar RD, Bez LG, Lopes CS. Experiência inicial com o uso de adesivo tissular contendo trombina para tratamento do pseudoaneurisma femoral. J Vasc Bras. 2006;5(1):30-6.

12. Krüger K, Zähringer $M$, Söhngen $F D$, Gossmann $A$, Schulte $O$, Feldmann $C$, et al. Femoral pseudoaneurysms: management with percutaneous thrombin injections: success rates and effects on systemic coagulation. Radiology. 2003;226(2):452-8.

13. Miranda FC, Garcia RG, Macedo Filho CL, Mendes GF, Andrade JR, Maurano A, et al. Injeção percutânea de trombina guiada por ultra-som com Doppler colorido para o tratamento de pseudo-aneurismas. Einstein. 2008;6(4):428-33.

14. Fellmeth BD, Roberts AC, Bookstein JJ, Freischlag JA, Forsythe JR, Buckner NK, et al. Postangiographic femoral artery injuries: nonsurgical repair with US-guided compression. Radiology. 1991;178(3):671-5.

15. Paulson EK, Nelson RC, Mayes CE, Sheafor DH, Sketch $\mathrm{MH} \mathrm{Jr}$, Kliewer MA. Sonographically guided thrombin injection of iatrogenic femoral pseudoaneurysm: further experience of a single institution. AJR Am J Roentgenol. 2001; 177(2):309-16.

16. Brophy DP, Sheiman RG, Amatulle P, Akbari CM. Iatrogenic femoral pseudoaneurysms: thrombin injection after failed US-guided compression. Radiology. 2000;214(1):278-82. 